\title{
Sinergismo microbiano entre óleos essenciais e conservantes sintéticos utilizados na
}

\section{indústria de alimentos}

\author{
Microbial synergism between essential oils and synthetic preservatives used in the food industry \\ Sinergismo microbiano entre aceites esenciales y conservantes sintéticos utilizados in la industria \\ alimentaria
}

Recebido: 04/02/2022 | Revisado: 14/02/2022 | Aceito: 16/02/2022 | Publicado: 23/02/2022

\author{
Matheus Péricles Silva Láscaris \\ ORCID: https://orcid.org/0000-0003-4071-5716 \\ Universidade Federal de Sergipe, Brasil \\ E-mail: matheus1709@hotmail.com \\ Carolina Natalie Fontes Aroxa \\ ORCID: https://orcid.org/0000-0002-4552-1657 \\ Universidade Federal de Sergipe, Brasil \\ E-mail: carolina_aroxa@outlook.com \\ Adrian Ramos Pio \\ ORCID: https://orcid.org/0000-0002-6525-4672 \\ Universidade Federal de Sergipe, Brasil \\ E-mail: adrianpio@outlook.com.br \\ Jenisson Linike Costa Gonçalves \\ ORCID: https://orcid.org/0000-0003-2136-2126 \\ Universidade Federal de Sergipe, Brasil \\ E-mail: jenissonlinike@gmail.com \\ Tatiana Pacheco Nunes \\ ORCID: https://orcid.org/0000-0001-9106-8622 \\ Universidade Federal de Sergipe, Brasil \\ E-mail: tpnunes@uol.com.br
}

\begin{abstract}
Resumo
O uso de aditivos alimentares é necessário para a distribuição de alimentos, porém devido à preocupação dos consumidores com a alimentação, a indústria alimentícia procura diminuir cada vez mais o uso de aditivos sintéticos, sem reduzir a qualidade de seus produtos. Sendo assim, esse trabalho objetivou avaliar o efeito antimicrobiano de óleos essenciais pelo método de disco-difusão, da concentração inibitória mínima (CIM) em microplacas e seu possível sinergismo com antimicrobianos sintéticos normalmente utilizados pela indústria pelo método "checkerboard". Dentre os óleos avaliados (Alecrim, Manjericão, Tangerina, Hortelã-Pimenta e Copaíba), o de Manjericão apresentou as maiores atividades antimicrobianas no teste de disco-difusão e o de Copaíba, as menores. $\mathrm{Na}$ análise de CIM observou-se que o de Alecrim e Copaíba foram mais eficazes contra L. monocytogenes e S. aureus $\left(0,31 \mathrm{mg} \cdot \mathrm{mL}^{-1}\right)$; Manjericão contra $S$. Typhimurium e L. monocytogenes $\left(0,62 \mathrm{mg} \cdot \mathrm{mL}^{-1}\right)$; Tangerina contra $L$. monocytogenes $\left(0,31 \mathrm{mg} \cdot \mathrm{mL}^{-1}\right)$ e Hortelã-Pimenta contra $B$. cereus $\left(0,62 \mathrm{mg} \cdot \mathrm{mL}^{-1}\right)$. Os antimicrobianos sintéticos foram efetivos contra todas as bactérias e variaram sua concentração entre 15 e $1,5 \mathrm{mg} \cdot \mathrm{mL}^{-1}$. Ao analisar o teste de sinergismo foi verificado que o óleo que mais apresentou associações predominantes sinérgicas foi o Manjericão, enquanto os outros óleos tiveram mais associações indiferentes ou aditivas. Sendo assim, a presente pesquisa conclui que há sinergismo in vitro entre os antimicrobianos sintéticos e os óleos essenciais, criando uma possibilidade para que a indústria de alimentos reduza o uso dos aditivos sintéticos aplicando essas associações em seus produtos.
\end{abstract}

Palavras-chave: Óleo essencial; Antimicrobiano sintético; Sinergismo.

\begin{abstract}
The use of food additives is necessary for the distribution of food, but due to the concern of consumers with food, the food industry increasingly seeks to reduce the use of synthetic additives, without reducing the quality of its products. Therefore, this work aimed to evaluate the antimicrobial effect of essential oils by the disk-diffusion method, the minimum inhibitory concentration (MIC) in microplates and their possible synergism with synthetic antimicrobials normally used by the industry by the "checkerboard" method. Among the evaluated oils (Rosemary, Basil, Tangerine, Peppermint and Copaíba), Basil showed the highest antimicrobial activities in the disk diffusion test and Copaíba, the lowest. In the MIC analysis, it was observed that Rosemary and Copaíba were more effective against $L$. monocytogenes and $S$. aureus $\left(0.31 \mathrm{mg} . \mathrm{mL}^{-1}\right)$; Basil against $S$. Typhimurium and L. monocytogenes $\left(0.62 \mathrm{mg} \cdot \mathrm{mL}^{-1}\right)$; Tangerine against $L$. monocytogenes $\left(0.31 \mathrm{mg} \cdot \mathrm{mL}^{-1}\right)$ and Peppermint against $B$. cereus $\left(0.62 \mathrm{mg} \cdot \mathrm{mL}^{-1}\right)$. Synthetic antimicrobials were effective against all bacteria and their concentration varied between 15 and $1.5 \mathrm{mg} \cdot \mathrm{mL}^{-1}$. When
\end{abstract}


analyzing the synergism test, it was verified that the oil that presented the most predominant synergistic associations was Basil, while the other oils had more indifferent or additive associations. Therefore, the present research concludes that there is in vitro synergism between synthetic antimicrobials and essential oils, creating a possibility for the food industry to reduce the use of synthetic additives by applying these associations in their products.

Keywords: Essential oil; Synthetic antimicrobial; Synergism.

\section{Resumen}

El uso de aditivos alimentarios es necesario para la distribución de alimentos, pero debido a la preocupación de los consumidores por los alimentos, la industria alimentaria busca cada vez más reducir el uso de aditivos sintéticos, sin mermar la calidad de sus productos. Este trabajo tuvo como objetivo evaluar el efecto antimicrobiano de los aceites esenciales por el método de difusión en disco, la concentración mínima inhibitoria (CMI) en microplacas y su posible sinergismo con los antimicrobianos sintéticos normalmente utilizados por la industria por el método "checkerboard". Los aceites evaluados (romero, albahaca, mandarina, menta piperita y copaíba), la albahaca mostró las actividades antimicrobianas más altas en la prueba de difusión en disco y Copaíba, la más baja. En el análisis de CMI se observó que Romero y Copaíba fueron más efectivos contra L. monocytogenes y $S$. aureus $\left(0,31 \mathrm{mg} \cdot \mathrm{mL}^{-1}\right)$; Albahaca contra $S$. Typhimurium y L. monocytogenes $\left(0.62 \mathrm{mg} \cdot \mathrm{mL}^{-1}\right)$; Mandarina contra L. monocytogenes $\left(0.31 \mathrm{mg} \cdot \mathrm{mL}^{-1}\right)$ y $\mathrm{Menta}$ contra B. cereus $\left(0.62 \mathrm{mg} \cdot \mathrm{mL}^{-1}\right)$. Los antimicrobianos sintéticos fueron efectivos contra todas las bacterias y su concentración varió entre 15 y 1,5 mg. $\mathrm{mL}^{-1}$. Al analizar la prueba de sinergismo se verificó que el aceite que presentó asociaciones sinérgicas más predominantes fue la Albahaca, mientras que los demás aceites presentaron asociaciones más indiferentes o aditivas. La presente investigación concluye que existe sinergismo in vitro entre los antimicrobianos sintéticos y los aceites esenciales, creando una posibilidad para la industria alimentaria de reducir el uso de aditivos sintéticos mediante la aplicación de estas asociaciones en sus productos.

Palabras clave: Aceite esencial; Antimicrobiano sintético; Sinergismo.

\section{Introdução}

Com os avanços da sociedade observa-se um aumento do consumo de alimentos processados, de conveniência e de prateleira e inevitavelmente elevou-se o consumo de aditivos alimentares, gerando uma preocupação na população quanto à ingestão exacerbada de produtos que contenham conservantes (Daltoé et al., 2020). A priori, os conservantes alimentares têm como função adiar ou impedir a ação de microrganismos e seu uso está relacionado a diversas características dos alimentos ( $\mathrm{pH}$, Aw, componentes nutricionais), como também o impacto no paladar, custo, eficácia e o tipo de microrganismo a ser combatido (Copetti, 2019).

É inquestionável a importância dos aditivos antimicrobianos para a geração de alimentos, pois os dados estatísticos das doenças transmitidas por alimentos (DTA) no Brasil, mostram que foram notificados no ano de 2017, 598 surtos de DTAs com 9426 enfermos, 1.439 hospitalizados e 12 óbitos (Ministério da Saúde, 2019). Nos Estados Unidos, o Centro de Controle e Prevenção de Doenças (CDC), estima que anualmente aproximadamente 48 milhões de casos são registrados, com 128 mil internações e 3 mil óbitos provocados por doenças causadas por alimentos (CDC, 2020).

Apesar da regulamentação existente pela ANVISA (Brasil, 1988), não se tem certeza sob as quantidades utilizadas pelas indústrias, pois não existe obrigatoriedade legal em declarar as quantidades presentes, somente a relação de aditivos utilizados. Porém, mesmo que os alimentos estejam dentro das normas estabelecidas pela ANVISA ou pelo Codex Alimentarius, a ingestão diária aceitável (IDA) não é avaliada, sendo assim a toxidade dessas substâncias a longo prazo é desconhecida, principalmente em crianças, visto que seu menor peso corpóreo, quando comparado com um adulto, provoca uma maior suscetibilidade às reações atribuladas provocadas pelos aditivos alimentares (Pereira et al., 2015).

Sendo assim, uma alternativa para a substituição ou redução do uso dos antimicrobianos sintéticos tradicionalmente utilizados é a utilização de óleos essenciais, que são compostos presentes naturalmente em plantas e especiarias, responsáveis por funções biológicas relacionadas aos seus mecanismos de defesa. São antimicrobianos naturais com potencial para serem usados como aditivos alimentares (Reis et al., 2020).

A atividade antimicrobiana de óleos essenciais de especiarias tem sido demonstrada em alimentos por diferentes estudos (Castorani \& Figueiredo, 2018; Freire et al., 2014; Monteiro, 2015) e, aliados à busca atual dos consumidores por 
produtos mais saudáveis e "naturais", esses compostos surgem como uma alternativa aos conservantes tradicionais. Essa atividade antimicrobiana vem da presença de álcoois, aldeídos e fenóis, porém em alguns casos acontece graças ao sinergismo de diferentes classes de compostos encontrados na sua composição (como fenóis, aldeídos, cetonas, álcoois, ésteres e hidrocarbonetos) (Bassolé et al., 2012; Bakkali, et al., 2008; Raut et al., 2014).

Entretanto, estudos indicam que o maior empecilho da indústria na introdução de óleos essenciais com foco na segurança dos alimentos são as altas concentrações, pois limitam a aceitabilidade sensorial dos produtos, cerca de $75 \%$ dos consumidores rejeitam um produto com odor/sabor forte de óleo essencial, sendo assim o uso apenas de óleo é inviável para a indústria, visto que apenas $25 \%$ indicam vontade de consumir esses produtos, sendo o sinergismo com antimicrobianos sintéticos já conhecidos uma possibilidade (García-Díez et al., 2016).

Esse sinergismo já foi observado por diversos autores, como Gutierrez et al. (2008) que relataram que os óleos essenciais quando combinados com outras substâncias podem ter o mesmo potencial antimicrobiano evidenciado quando utilizados isoladamente, contudo, em menores concentrações. Os estudos realizados por Zago et al. (2009) deram origem à possibilidade de utilização de óleos essenciais combinados aos antimicrobianos tradicionais, com um aumento no potencial antimicrobiano. Os autores relataram que mesmo óleos essenciais que não demonstraram muita eficiência na inibição do crescimento microbiano, ao serem associados a produtos sintéticos, apresentaram sinergismo.

Sendo assim, esse trabalho teve como objetivo avaliar o potencial antimicrobiano já conhecido de óleos essenciais utilizados em aromaterapia, bem como o possível sinergismo com conservantes químicos utilizados pela indústria de alimentos.

\section{Metodologia}

Nesse estudo foi utilizada metodologia quantitativa conforme explicado por Pereira et al. (2018).

\section{Óleos essenciais, aditivos alimentares e cepas bacterianas}

Os óleos essenciais de Alecrim (Lote: 2029311X), Manjericão (Lote: 2023310X), Tangerina (Lote: 2211601BR), Copaíba (Lote: 202615X) e Hortelã-Pimenta (Lote: 2212140BR) foram obtidos da empresa DoTERRA. Os antimicrobianos sintéticos Metabissulfito de Sódio (GRILLO-WERKAG, lote:GFI590608), Sorbato de Potássio (NINGBO WANGLONG TECH CO, LTD, lote: 1032005102) e Benzoato de Sódio (Tianjin Dongda Chemical Group CO, LTD, lote: 20191223) foram gentilmente cedidos pela Cooperativa Agroindustrial dos Irrigantes do Projeto Bananeira (COOPAIBA/SE) e as cepas bacterianas utilizadas para avaliar a atividade antimicrobiana foram: Staphylococcus aureus (ATCC 6538), Bacillus cereus (ATCC 11778), Listeria monocytogenes (ATCC 7644), Escherichia coli (ATCC 8739), Salmonella Enteritidis (ATCC 13076) e Salmonellla Typhimurium (ATCC 14028).

\section{Preparo das soluções e inóculo bacteriano}

As soluções de óleos essenciais foram preparadas em eppendorfs estéreis. Para o preparo dessa solução foi adicionado $1 \mathrm{~mL}$ de dimetilsulfoxido (DMSO) e $10 \mathrm{mg}$ de óleo essencial, resultando em uma solução com concentração de $10 \mathrm{mg} \cdot \mathrm{mL}^{-1}$ de óleo essencial. As soluções de antimicrobianos sintéticos foram preparados em água destilada nas concentrações de 30 mg.mL ${ }^{1}$ para o Metabissulfito de Sódio (BRASIL, 2013) e $20 \mathrm{mg} \cdot \mathrm{mL}^{-1}$ para o Sorbato de Potássio e Benzoato de Sódio (BRASIL, 2013).

Os inóculos bacterianos, para o método de difusão em disco, foram cultivados em caldo Brain Heart Infusion (KASVI) com densidades ajustadas para coincidir com o padrão de turvação de 0,5 unidades de Mc Farland (10 ${ }^{8}$ UFC.mL $\left.{ }^{-1}\right)$. 
Os inóculos para o teste de concentração inibitória mínima (CIM) e para o método de "checkerboard" de sinergismo foram preparados da mesma maneira para os óleos e o antimicrobiano Metabissulfito de Sódio. Para os antimicrobianos Sorbato de Potássio e Benzoato de Sódio os microrganismos foram cultivados em caldo Brain Heart Infusion (KASVI) acidificado com ácido clorídrico P.A (NEON) até pH 5, com densidades ajustadas para coincidir com o padrão de turvação de 0,5 unidades de Mc Farland $\left(10^{8}\right.$ UFC.mL $\left.{ }^{-1}\right)$.

\section{Método de difusão em disco}

Para a confirmação da atividade antimicrobiana dos óleos essenciais foi escolhido o método de disco-difusão proposto por Jing-En et al. (2015), no qual $100 \mu \mathrm{L}$ da suspensão bacteriana foram semeados em placas contendo $20 \mathrm{~mL}$ de Agar Mueller Hilton (KASVI), previamente preparado, esterilizado, dispensado sobre as mesmas e solidificado. Após a semeadura, cinco discos de papel de filtro de $5 \mathrm{~mm}$ foram alocados na superfície do meio, no qual um disco foi adicionado do controle positivo (clorexidina 0,12\%), e um do controle negativo (DMSO) e em três deles foram adicionados de $10 \mu \mathrm{L}$ das soluções de óleos essenciais. A zona de inibição (ZI) do crescimento microbiano foi avaliada pelo diâmetro do halo de inibição em mm ao redor dos discos após 24 horas de incubação a $37 \pm 1{ }^{\circ} \mathrm{C}$. Os ensaios foram realizados em triplicatas, e os resultados expressos pela média aritmética dos valores dos halos obtidos nas repetições.

\section{Determinação da Concentração Inibitória Mínima (CIM)}

O teste da Concentração Inibitória Mínima (CIM) seguiu conforme protocolo proposto pelo National Committee for Clinical Laboratory Standards (NCCLS, 2003). Para análise do CIM foram utilizadas microplacas de poliestireno estéreis de 96 poços. Foram adicionados $100 \mu \mathrm{L}$ de caldo BHI em concentração dupla em todos os poços da placa. O óleo essencial ou o antimicrobiano sintético testado foi adicionado à primeira linha de poços da microplaca. Em seguida, foram realizadas diluições seriadas das soluções, sendo que o poço seguinte apresentou a metade da concentração do poço imediatamente anterior. Foram inoculados $10 \mu \mathrm{L}$ da suspensão bacteriana em cada poço das respectivas microplacas, com exceção da fileira onde foi feito o controle da esterilidade do caldo. As microplacas foram incubadas a $35^{\circ} \mathrm{C}$, por 24 horas. Após este período, foram adicionados $20 \mu \mathrm{L}$ de TTC (Trifeniltetrazolio-2,3,5 Cloreto) a 0,5\% (v/v) em cada poço e em seguida as placas foram reincubadas por mais 2 horas. A mudança de incolor para coloração vermelha indicou a presença de microrganismos, e assim a menor concentração para a qual não houve crescimento das bactérias foi definida como a CIM. O valor CIM foi registrado como a concentração média da triplicata.

\section{Associação do óleo essencial com conservante químico}

Os óleos essenciais e os antimicrobianos sintéticos que apresentaram ação antimicrobiana foram submetidos ao teste de sinergismo entre si, através da técnica de "checkerboard”, para derivação do índice de concentração inibitória fracionada. Foram utilizadas as soluções de óleos essenciais de acordo com as respectivas CIM. Primeiramente, foram adicionados às microplacas estéreis de 96 cavidades $100 \mu \mathrm{L}$ de caldo BHI estéril em concentração dupla. Posteriormente, foram transferidas alíquotas de $100 \mu \mathrm{L}$ das soluções de óleo essencial a serem testadas nos sete primeiros poços da primeira linha, na concentração de 8xCIM, onde foi diluída no sentido vertical, de modo a ter concentrações nas colunas com os respectivos valores de CIMx8, CIMx4, CIMx2, CIM, CIM/2, CIM/4, CIM/8.

Em seguida, foram adicionados, da primeira coluna até a sétima, $100 \mu \mathrm{L}$ da solução conservante, mantendo a concentração fixa no sentido vertical, sendo que do primeiro poço até o último poço da primeira coluna foi adicionado a solução de conservante químico CIM/8, até CIMx8 na última coluna de óleo essencial, respectivamente. Por fim, foram 
adicionadas alíquotas de $10 \mu \mathrm{L}$ da suspensão bacteriana já padronizada, nos poços das microplacas. Estas foram incubadas por 24 horas a $35^{\circ} \mathrm{C}$.

Para o cálculo do índice de concentração inibitória fracionada (ICIF), foi realizada a soma da concentração inibitória fracionada de A (CIFA) + concentração inibitória fracionada de B (CIFB), sendo que A representa o óleo essencial e B o conservante químico. A determinação de CIFA foi feita a partir da divisão da concentração inibitória mínima no teste de “checkerboard" (CIM-A combinada) pela concentração inibitória mínima (CIM-A sozinha), assim como CIFB foi obtida através do quociente de CIM-B combinada por CIM-B sozinha. Dessa forma, a classificação do ICIF de acordo com a atividade dos óleos conjunta ao conservante químico foi classificada em sinérgica $(\leq 0,5)$, aditiva (entre 0,5 e 1,0$)$, indiferente (entre 1,0 e 4,0) ou antagônica (>4,0) (Lewis et al., 2002).

\section{Análise Estatística}

Todas as análises foram realizadas em triplicata e os resultados foram expressos pela média aritmética dos valores obtidos nas três repetições. Esses resultados foram submetidos à análise de variância e teste de Tukey (p<0,05), pelo programa gratuito Sisvar.

\section{Resultados e Discussão}

\subsection{Teste de disco-difusão}

Na Tabela 1 encontram-se os resultados referentes à análise de disco-difusão dos óleos essenciais.

Tabela 1. Atividade antimicrobiana dos óleos essenciais pelo método disco-difusão em mm.

\begin{tabular}{|c|c|c|c|c|c|c|}
\hline \multirow[b]{2}{*}{ Microrganismo } & \multicolumn{5}{|c|}{ Óleos Essenciais (10mg.mL-1) } & \multirow[b]{2}{*}{ Controle +} \\
\hline & Alecrim & Manjericão & Tangerina & $\begin{array}{l}\text { Hortelã- } \\
\text { Pimenta }\end{array}$ & Copaíba & \\
\hline \multicolumn{7}{|l|}{ Salmonella } \\
\hline Typhimurium & $11,16 \pm 1,02^{\mathrm{a}}$ & $10,83 \pm 1,17^{\mathrm{a}}$ & $10,33 \pm 0,23^{\mathrm{a}}$ & $11,66 \pm 2,01^{\mathrm{a}}$ & $10,00 \pm 0,40^{\mathrm{a}}$ & $14,50 \pm 0,81^{\mathrm{b}}$ \\
\hline Salmonella Enteritidis & $10,00 \pm 0,40^{\mathrm{a}}$ & $14,50 \pm 0,40^{\mathrm{b}}$ & $10,00 \pm 0,40^{\mathrm{a}}$ & $13,00 \pm 1,63^{b}$ & $9,83 \pm 0,23^{\mathrm{a}}$ & $14,16 \pm 0,84^{b}$ \\
\hline Escherichia coli & $10,75 \pm 0,35^{b}$ & $11,33 \pm 0,23^{\mathrm{b}}$ & $10,00 \pm 0,40^{\mathrm{ab}}$ & $12,16 \pm 0,94^{\mathrm{b}}$ & $8,00 \pm 0,70^{\mathrm{a}}$ & $11,83 \pm 1,88^{b}$ \\
\hline Listeria monocytogenes & $10,50 \pm 0,40^{\mathrm{b}}$ & $15,16 \pm 0,47^{\mathrm{c}}$ & $10,83 \pm 0,23^{\mathrm{b}}$ & $11,00 \pm 1,08^{b}$ & $7,00 \pm 0,81^{\mathrm{a}}$ & $11,50 \pm 0,70^{\mathrm{b}}$ \\
\hline Staphylococcus aureus & $10,33 \pm 0,62^{\mathrm{a}}$ & $17,50 \pm 0,81^{\mathrm{c}}$ & $10,16 \pm 0,23^{\mathrm{a}}$ & $12,00 \pm 0,40^{\mathrm{a}}$ & $10,16 \pm 0,23^{a}$ & $14,83 \pm 0,84^{\mathrm{b}}$ \\
\hline Bacillus cereus & $10,83 \pm 0,62^{b}$ & $16,50 \pm 0,81^{\mathrm{c}}$ & $9,83 \pm 0,23^{b}$ & $14,16 \pm 0,62^{\mathrm{c}}$ & $0 \pm 0^{\mathrm{a}}$ & $14,66 \pm 0,23^{\mathrm{c}}$ \\
\hline
\end{tabular}

As análises foram realizadas em triplicata e os resultados estão expressos pela média \pm desvio padrão. Letras iguais na mesma linha não diferem significativamente entre si $(\mathrm{p}<0,05)$, segundo teste Tukey. Fonte: Autores (2022).

Na Tabela 1 pode se observar que todos os óleos apresentaram atividade antimicrobiana contra os microrganismos testados, exceto o óleo de copaíba para o Bacillus cereus. Segundo Mothana e Lingequist (2005), os óleos que apresentam halos de inibição entre 8 e 13 mm são classificados como atividade moderada e os halos maiores que 14mm são classificados como atividade alta, que foi o caso apenas do óleo de Manjericão contra Salmonella Enteritidis, Listeria monocytogenes, Staphylococcus aureus e Bacillus cereus, e o óleo de Hortelã-Pimenta contra o último microrganismo citado. Todos os demais óleos analisados apresentaram atividade moderada (Tabela 1). Esses resultados são concordantes aos relatados por Cutrim e 
seus colaboradores (2019), que também avaliaram a atividade antimicrobiana do óleo de Alecrim e o classificaram como atividade moderada, já que reportaram halos com 10,7 e 9,7 mm para cepas de $S$. aureus e E. coli, respectivamente, similar ao encontrado nesse estudo (10,30 e 10,75 mm).

Ao analisar o óleo de Manjericão, ele apresentou os melhores resultados para $S$. Enteritidis $(14,50 \mathrm{~mm}), L$. monocytogenes $(15,16 \mathrm{~mm}), S$. aureus $(17,50 \mathrm{~mm})$ e B. cereus $(16,50 \mathrm{~mm})$, sendo classificado com alta atividade antimicrobiana, superando até mesmo o antibiótico clorexidina $0,12 \%$, utilizado como controle positivo. Mendonça (2018) estudou diversos cultivares de manjericão e reportou que todos eles apresentavam ação antibacteriana contra $S$. aureus, $B$. cereus, L. monocytogenes, E. coli e S. Typhimurium, assim como Silva \& Mello (2021) que encontraram um halo de $14 \mathrm{~mm}$ para S. aureus com o mesmo tipo de óleo.

O óleo de Tangerina, assim como o de Alecrim, mostrou-se com atividade antimicrobiana moderada, já que apresentaram halos variando entre $9,83 \mathrm{~mm}$ (B. cereus) e 10,83 mm (L. monocytogenes). Por outro lado, Santos et al. (2016) relataram valores inferiores aos encontrados nessa pesquisa, com halos de inibição de 4,88 mm para $S$. aureus, 5,11 mm para E. coli e de 5,38 mm para Salmonella spp., sendo classificado com um óleo de baixa atividade antimicrobiana (< $8 \mathrm{~mm})$. A diferença observada entre esses resultados pode ser derivada de vários fatores, pois cada óleo apresenta uma composição diferente mesmo tendo origem na mesma matéria prima, devido a sua localização geográfica, estágio de maturação do fruto e método de extração (Spisni et al., 2020).

Pode-se verificar que o óleo de Hortelã-Pimenta apresentou alta atividade contra a cepa de B. cereus $(14,16 \mathrm{~mm}) \mathrm{e}$ atividade moderada contra todas as outras cepas, tendo resultados entre 11 e $13 \mathrm{~mm}$. Silva et al. (2019) estudaram a atividade antimicrobiana desse mesmo óleo de cinco diferentes empresas e observaram que todos apresentaram atividade contra $S$. aureus e E. coli.

O óleo de Copaíba apresentou os menores valores para todas as cepas testadas. Observou-se baixa atividade antimicrobiana contra L. monocytogenes $(7,0 \mathrm{~mm})$ e a não formação de halo com o B. cereus $(0,0$ mm), verificando a maior atividade contra $S$. aureus $(10,16 \mathrm{~mm})$. No entanto, Pereira et al. (2018) reportaram alta atividade analisando óleo comercial de copaíba para E. coli $(17,6 \mathrm{~mm})$ e para $S$. aureus $(11,7 \mathrm{~mm})$. Outros autores reforçam que a variedade dos compostos presentes nos óleos pode ser derivada do clima, do tipo de cultivo, da forma de extração e armazenamento podendo aumentar ou diminuir o potencial antimicrobiano deles (Santos et al., 2016).

Apesar dos bons resultados, o método de difusão em disco não é respaldo para a determinação da concentração inibitória mínima, pois apresenta fatores que interferem na difusão dos compostos no meio de cultura (espessura do ágar, volume, propriedades físico-químicas, hidrossolubilidade, massa molecular e características lipofílicas das amostras), sendo a diluição em caldo a técnica mais adequada para determinar a atividade antimicrobiana (Amparo et al., 2018; Alves et al., 2008).

\subsection{Concentração inibitória mínima dos óleos essenciais e dos antimicrobianos sintéticos}

Os resultados obtidos da análise da concentração inibitória mínima dos óleos essenciais estão dispostos na Tabela 2. 
Tabela 2. Resultados da Análise de Concentração Inibitória Mínima (CIM) dos óleos essenciais, realizado em triplicata.

\begin{tabular}{|c|c|c|c|c|c|}
\hline \multirow[b]{2}{*}{ Microrganismo } & \multicolumn{5}{|c|}{ Óleos (mg.mL $\left.L^{-1}\right)$} \\
\hline & Alecrim & Manjericão & Tangerina & $\begin{array}{l}\text { Hortelã- } \\
\text { Pimenta }\end{array}$ & Copaíba \\
\hline Salmonella Typhimurium & 1,25 & 0,62 & 1,25 & 1,25 & 5 \\
\hline Salmonella Enteritidis & 1,25 & 1,25 & 1,25 & 1,25 & 5 \\
\hline Escherichia coli & 1,25 & 1,25 & 1,25 & 2,50 & 5 \\
\hline Listeria monocytogenes & 0,62 & 0,62 & 0,31 & 2,50 & 0,31 \\
\hline Staphylococcus aureus & 0,62 & 1,25 & 0,62 & 2,50 & 0,31 \\
\hline Bacillus cereus & 2,50 & 1,25 & 2,50 & 0,62 & - \\
\hline
\end{tabular}

Legenda: - não houve inibição. Fonte: Autores (2022).

Aligianis et al. (2001) classificaram a atividade antimicrobiana para materiais vegetais, com relação a concentração inibitória mínima, como: Inibição forte até $0,5 \mathrm{mg} \cdot \mathrm{mL}^{-1}$; Inibição moderada entre 0,6 e $1 \mathrm{mg} \cdot \mathrm{mL}^{-1}$; Inibição fraca acima de 1 mg.mL $\mathrm{L}^{-1}$. O estudo da CIM é importante para avaliar a resistência do microrganismo ao óleo testado.

Sendo assim, na Tabela 2, é possível perceber que o óleo de Alecrim possui inibição fraca contra $B$. cereus $\left(2,5 \mathrm{mg} . \mathrm{mL}^{-1}\right), S$. Typhimurium, $S$. Enteritidis e E. coli $\left(1,25 \mathrm{mg} . \mathrm{mL}^{-1}\right.$ para todos) e inibição moderada para L. monocytogenes e $S$. aureus $(0,62$ mg. $\mathrm{mL}^{-1}$ ). Outros autores também encontraram inibição fraca e moderada para esse óleo, como Silva et al. (2015), que reportaram valores de CIM maiores que $1 \mathrm{mg} \cdot \mathrm{mL}^{-1}$ para E. coli e S. aureus e Silva et al. (2018) que relataram valores de CIM de 4,39 , 2,27 e 3,29 mg.mL $\mathrm{m}^{-1}$ para E. coli, S. aureus e Salmonella ssp., respectivamente.

O óleo de Manjericão, apesar de demonstrar os melhores resultados na análise de disco-difusão, apresentou inibição fraca para $S$. Enteritidis, E. coli, $S$. aureus e B. cereus, com CIM de 1,25 mg.mL-1, e uma inibição moderada contra $S$. Typhimurium e L. monocytogenes (0,62 mg. $\left.\mathrm{mL}^{-1}\right)$. Mendonça (2018) em seu estudo com óleos de manjericão extraídos de diversos cultivares, também reportou CIM de 0,62 mg.mL $\mathrm{mL}^{-1}$ para L. monocytogenes e $S$. Typhimurium, bem como CIM de 1,25 mg.mL $L^{-1}$ para cepas de S. aureus. Por outro lado, Oliveira (2015), ao avaliar a incorporação desse óleo em biofilmes relatou uma inibição fraca frente às cepas de $E$. coli e $S$. aureus $\left(5 \mathrm{mg} \cdot \mathrm{mL}^{-1} \mathrm{e} 2,5 \mathrm{mg} \cdot \mathrm{mL}^{-1}\right.$, respectivamente), provavelmente devido à fraca interação dos compostos do óleo com os polímeros presentes no biofilme.

Observando o óleo de Tangerina, verifica-se uma inibição forte contra L. monocytogenes $\left(0,31 \mathrm{mg} \cdot \mathrm{mL}^{-1}\right)$ e moderada contra $S$. aureus $\left(0,62 \mathrm{mg} \cdot \mathrm{mL}^{-1}\right)$. Com os demais microrganismos apresentou uma inibição fraca, para $S$. Typhimurium, $S$. Enteritidis, E. coli 1,25 mg.mL $\mathrm{mL}^{-1}$ e para B. cereus 2,50 mg. $\mathrm{mL}^{-1}$. Por outro lado, Carvalho (2016) não observou atividade antimicrobiana desse óleo em seu estudo, enquanto Santos et al. (2016) estudaram a eficácia de diferentes óleos essenciais cítricos e reportaram que o CIM foi de $80 \mathrm{uL} \cdot \mathrm{mL}^{-1}$ para E. coli, $40 \mathrm{uL} . \mathrm{mL}^{-1}$ para Salmonella ssp. e 2 uL.mL ${ }^{-1}$ para $S$. aureus, valores inferiores ao encontrados na presente pesquisa.

Verificou-se que o óleo de Hortelã-pimenta foi o que as bactérias apresentaram maior resistência, exceto o $B$. cereus $\left(0,62 \mathrm{mg} \cdot \mathrm{mL}^{-1}\right)$. Outros autores estudaram esse óleo essencial frente a outros microrganismos e obtiveram uma inibição fraca, como Chouhan et al., (2017), que determinou a CIM de 10mg. $\mathrm{mL}^{-1}$ contra Clostridium perfringens e Rodrigues (2017) que estudou esse óleo contra cepas de bactérias Actinobacillus pleuropneumoniae, encontrando também uma inibição fraca. 
O óleo de Copaíba, assim como no teste de disco-difusão, apresentou os piores resultados para as bactérias gramnegativas, uma inibição fraca, com CIM de $5 \mathrm{mg} \cdot \mathrm{mL}^{-1}$ e não apresentou eficácia contra $B$. cereus, porém, $L$. monocytogenes e $S$. aureus apresentaram uma sensibilidade ao óleo, tendo uma CIM de 0,31 mg. $\mathrm{mL}^{-1}$ (uma inibição forte). Essa sensibilidade do $S$. aureus frente ao óleo de copaíba já foi reportado por Guimarães et al. (2016) (0,31 mg.mL $\left.{ }^{-1}\right)$, bem como por Masson et al. (2013) $\left(0,2 \mathrm{mg} \cdot \mathrm{mL}^{-1}\right)$.

Na Tabela 3 é possível verificar a concentração inibitória mínima dos aditivos utilizados tradicionalmente pela indústria de alimentos (Metabissulfito de Sódio, Sorbato de Potássio e Benzoato de Sódio).

Tabela 3. Resultados da Análise da Concentração Inibitória Mínima dos antimicrobianos sintéticos, realizados em triplicata.

\begin{tabular}{lccc}
\hline & \multicolumn{3}{c}{ Antimicrobianos Sintéticos (mg.mL $\left.\mathbf{m}^{-\mathbf{1}}\right)$} \\
\cline { 2 - 4 } Microrganismo & 15 & Sorbato de Potássio & Benzoato de Sódio \\
\hline Salmonella Typhimurium & 15 & 1,25 & 2,5 \\
Salmonella Enteritidis & 15 & 20 & 2,5 \\
Escherichia coli & 30 & 2,5 & 1,25 \\
Listeria monocytogenes & 15 & 1,25 & 2,5 \\
Staphylococcus aureus & 15 & 5 & 5 \\
Bacillus cereus & & 2,5 & 1,25 \\
\hline
\end{tabular}

Fonte: Autores (2022).

Observa-se na Tabela 3 é possível observar que todos os antimicrobianos sintéticos inibiram as bactérias testadas. O grupo dos sulfitos, presente no Metabissulfito, apresenta um mecanismo de ação que causa a ruptura da membrana citoplasmática e inativa a replicação do DNA e das enzimas a ela vinculadas a membrana ou a reações metabólicas, inativando o microrganismo independente da composição da parede celular (Favero et al., 2011). O Metabissulfito de Sódio apresentou o mesmo valor para quase todos os microrganismos $\left(15 \mathrm{mg} \cdot \mathrm{mL}^{-1}\right)$, exceto para L. monocytogenes, que foi mais resistente, necessitando de $30 \mathrm{mg} \cdot \mathrm{mL}^{-1}$ para ser inibida, corroborando com Jay (2005) que indica que a ação desse componente ocorre acima de 0,2 mg.mL $\mathrm{mL}^{-1}$. Ladeira et al. (2017) determinaram 0,2 mg.mL-1 em seu estudo contra $S$. Enteritidis e Ramos et al. (2015) verificaram CIM de 1,87 mg. $\mathrm{mL}^{-1}$ para B. cereus, E. coli, S. Typhimuriume S. aureus, todos valores abaixo aos encontrados na presente pesquisa, mas dentro do que preconiza a literatura.

Em relação ao Sorbato de Potássio, S. Typhimurium e L. monocytogenes foram os mais sensíveis a esse antimicrobiano $\left(1,25 \mathrm{mg} \cdot \mathrm{mL}^{-1}\right)$, enquanto $S$. Enteritidis foi o mais resistente $\left(20 \mathrm{mg} \cdot \mathrm{mL}^{-1}\right)$. O mecanismo de ação desse conservante ainda não é muito conhecido, porém estudos indicam que ocorre a acidificação intracelular dos microrganismos ou o estresse celular, aumentando a produção de toxinas (Mira et al., 2010; Fodil et al., 2018). Ferreira et al. (2020) estudaram diversos antimicrobianos sintéticos contra a $S$. Typhimurium e reportaram valores maiores de CIM quanto comparado à presente pesquisa, entretanto Ferreira e colaboradores avaliaram o efeito do Sorbato de Potássio em pH 7, sendo um pH não adequado para o uso desse antimicrobiano, pois Segundo Wang et al. (2018), em pH mais próximo de 7 a ação antimicrobiana dos sorbatos diminuem, pois apenas a forma não ionizada consegue penetrar nas células.

O Benzoato de Sódio apresentou os melhores resultados, sendo que inibiu todos os microrganismos com concentrações menores ou iguais a $5 \mathrm{mg} \cdot \mathrm{mL}^{-1}$, sendo $S$. aureus o mais resistente $\left(5 \mathrm{mg} \cdot \mathrm{mL}^{-1}\right)$ e B. cereus e E. coli os mais sensíveis $\left(1,25 \mathrm{mg} \cdot \mathrm{mL}^{-1}\right)$. O mecanismo de atuação desse composto é a sua conversão em ácido benzóico acidificando o meio, 
dessa forma as bactérias precisam liberar prótons extras para o meio extracelular, consumindo ATP e levando a danos e/ou morte celular, e assim como os sorbatos, os benzoatos são mais eficazes em pH ácido (Chen \& Zhong, 2018). Ferreira e seus colaboradores (2020) também verificaram a eficácia do Benzoato de Sódio contra $S$. Typhimurium e encontraram 2,3 mg.mL ${ }^{-1}$, valor bem próximo ao desse estudo.

A legislação brasileira preconiza a quantidade máxima de aditivos em diversos alimentos e esse valor é variado de acordo com as características dos mesmos. Os antimicrobianos aqui estudados são utilizados principalmente em alimentos de origem vegetal e devem seguir a $\operatorname{RDC~}^{\circ}$ 8, de 6 de março de 2013 (Brasil, 2013). Vale ressaltar que a legislação expressa o limite de acordo com o composto ativo do conservante, não o conservante em si, por isso esse valor varia de acordo com as características do alimento que é aplicado (Ferreira et al., 2020).

\subsection{Sinergismo entre os óleos essenciais e os antimicrobianos sintéticos}

Com os dados da CIM dos óleos e dos antimicrobianos pode-se calcular índice de concentração inibitória fracionada (ICIF), que corresponde ao sinergismo entre as substâncias. Lewis e seus colaboradores (2002), classificaram a ICIF de maneira que $\leq$ 0,5 é classificado como sinérgica; 0,5 a 1 aditiva; 1 a 4 indiferente e > 4,0 antagônica.

$\mathrm{Na}$ Tabela 4 podemos verificar se ocorreu sinergismo ou não entre os óleos essenciais e os antimicrobianos sintéticos frente as bactérias Salmonella Typhimurium, Salmonella Enteritidis, Escherichia coli, Listeria monocytogenes, Staphylococcus aureus e Bacillus cereus.

É possível verificar, nessa tabela, todas as possibilidades de sinergismo. As combinações que apresentaram sinergismo (S) significam que houve uma redução significativa do óleo e do conservante sintético. As combinações que apresentaram ação aditiva (A) representam que houve redução somente do óleo ou do conservante sintético. As combinações onde se tem indiferença (-) indicam que não houve redução da concentração mínima inibitória do óleo ou do conservante e as combinações que aconteceu antagonismo $(\mathrm{X})$ significam que precisou aumentar a concentração do óleo ou do conservante para ocorresse a inibição da bactéria.

Tabela 4. Sinergismo entre os óleos essenciais com os antimicrobianos sintéticos.

\begin{tabular}{|c|c|c|c|c|c|c|c|c|c|c|c|c|c|c|c|}
\hline \multirow[t]{3}{*}{ Bactérias } & \multicolumn{15}{|c|}{ Óleos } \\
\hline & \multicolumn{3}{|c|}{ Alecrim } & \multicolumn{3}{|c|}{ Manjericão } & \multicolumn{3}{|c|}{ Tangerina } & \multicolumn{3}{|c|}{ Hortelã-Pimenta } & \multicolumn{3}{|c|}{ Copaíba } \\
\hline & MS & SP & BS & MS & SP & BS & MS & SP & BS & MS & SP & BS & MS & SP & BS \\
\hline S. Typhimurium & - & $\mathrm{S}$ & $\mathrm{S}$ & A & $S$ & $S$ & - & A & $\mathrm{S}$ & S & $\mathrm{S}$ & $\mathrm{S}$ & $S$ & - & $\mathrm{S}$ \\
\hline S.Enteritidis & A & $\mathrm{S}$ & $\mathrm{S}$ & $S$ & A & $\mathrm{S}$ & $S$ & S & $\mathrm{S}$ & S & $\mathrm{S}$ & A & $S$ & $S$ & A \\
\hline E. coli & A & - & $\mathrm{A}$ & $S$ & S & A & - & A & A & $\mathrm{S}$ & $\mathrm{S}$ & $\mathrm{S}$ & A & A & A \\
\hline L. monocytogenes & A & - & $\mathrm{X}$ & - & $S$ & $S$ & A & - & - & $S$ & A & A & $\mathrm{X}$ & - & - \\
\hline S. aureus & - & $\mathrm{S}$ & $\mathrm{S}$ & $S$ & $S$ & $S$ & $X$ & A & $S$ & $\mathrm{~S}$ & $\mathrm{~S}$ & $\mathrm{~S}$ & - & - & A \\
\hline B. cereus & A & $\mathrm{S}$ & - & A & A & - & - & A & - & A & - & - & $\mathrm{n} / \mathrm{d}$ & $\mathrm{n} / \mathrm{d}$ & $\mathrm{n} / \mathrm{d}$ \\
\hline
\end{tabular}

Legenda: MS= Metabissulfito de Sódio; SP= Sorbato de Potássio; BS= Benzoato de Sódio; S= Sinergismo; A= Ação aditiva; - = Indiferente; $\mathrm{X}=$ Antagônico; n/d= Não determinado. Fonte: Autores (2022). 
O óleo de Alecrim não apresentou sinergismo com o metabissulfito, porém apresentou com o sorbato para $S$. Typhimurium, $S$. Enteritidis, S. aureus e B.cereus. Por outro lado, foi observado efeito antagônico com o benzoato para $L$. monocytogenes, indiferente para B. cereus, aditivo para E. coli e sinérgico para as demais bactérias.

Na associação do óleo de Manjericão com o metabissulfito observou-se sinergismo para $S$. Enteritidis, $E$. coli e $S$. aureus. Quando associado ao benzoato continuou se observando o sinergismo para as mesmas bactérias além de $L$. monocytogenes, porém ao ser associado ao sorbato, apresentou ação aditiva para S. Enteritidis. Enirger et al. (2017) estudou a associação do antimicrobiano metabissulfito com óleo de orégano frente a B. cereus, S. aureus e E. coli, onde encontrou ação aditiva para todos os microrganismos, associação semelhante à encontrado nessa pesquisa para os óleos de alecrim, manjericão e hortelã-pimenta para B. cereus, ao óleo de tangerina para S. aureus e aos de alecrim e copaíba para E. coli.

A associação do óleo de Tangerina com os três antimicrobianos foi bem-sucedida para $S$. Enteritidis, porém a associação desse óleo ao metabissulfito mostrou-se antagônica para $S$. aureus, indiferente para $S$. Typhimurium, E. coli e $B$. cereus e aditiva para L. monocytogenes. Ao analisar essa associação com o benzoato também foi verificado efeito sinérgico para as cepas de $S$. Typhimurium e $S$. aureus, entretanto efeito aditivo para E. coli e indiferente para L. monocytogenese, B. cereus. O óleo de Hortelã-Pimenta foi o que apresentou a maior quantidade de efeito sinérgico com os antimicrobianos sintéticos, não apresentando esse efeito apenas para B. cereus (aditivo e indiferente) independente do conservante sintético. Ramos et al. (2015), ao associarem metabissulfito e óleo essencial de Tomilho reportaram resultados semelhantes ao óleo de Hortelã-Pimenta desse estudo, pois também observaram efeito sinérgico para B. cereus, E. coli, S. Typhimuriume S. aureus.

O óleo de Copaíba apresentou sinergismo apenas para as associações com metabissulfito frente às duas cepas de Salmonella, sorbato contra $S$. Enteritidis e benzoato contra $S$. Typhimurium, enquanto as outras associações foram ou aditivas ou indiferentes. Ekhtelat et al. (2019) estudaram o aditivo benzoato junto aos óleos essenciais de hortelã-silvestre, hortelãverde e cominho e verificaram a existência de sinergismo para as cepas de E. coli 0157:H7 e L. monocytogenes, sendo o mesmo resultado para a associação deste antimicrobiano com óleo de manjericão para a L. monocytogenes observado no presente estudo.

Vale ressaltar que a diferença entre a ação sinérgica, aditiva, antagônica ou indiferente observada entre os óleos e os conservantes sintéticos na presente pesquisa pode ser justificada pela presença das diferentes classes de compostos antimicrobianos que compõe a matéria-prima do óleo essencial, bem como suas interações com os conservantes sintéticos, resultando em uma atuação distinta no metabolismo celular dos microrganismos testados.

\section{Considerações Finais}

Diante dos resultados obtidos nessa pesquisa, podemos verificar que os óleos essenciais continuam sendo uma ótima opção para garantir a segurança de alimentos, entretanto não se deve descartar o estudo da associação com os conservantes sintéticos, pois o fator sensorial ainda é um problema relatado pela indústria para a aplicação desses compostos como aditivos nos alimentos. Sendo assim, o óleo de Alecrim e de Copaíba necessitaram de uma concentração menor para inibir $L$. monocytogenes e S. aureus, enquanto o óleo de Manjericão inibiu S. Typhimurium e L. monocytogenes em sua menor concentração. O óleo de Hortelã-pimenta teve sua menor concentração eficaz somente contra $B$. cereus, e o de Tangerina apenas para L. monocytogenes. No teste de sinergismo foram verificados poucos casos de antagonismos dentre os microrganismos testados, predominando o efeito aditivo, dentre as combinações possíveis a que mais apresentou sinergismo foi o óleo de Hortelã-Pimenta com Metabissulfito de Sódio, o Sorbato de Potássio associado ao óleo de Alecrim, Manjericão e Hortelã-Pimenta, bem como o óleo de Manjericão com o Benzoato de Sódio. Assim, vemos que o sinergismo entre esses compostos é possível e uma ótima alternativa para a indústria de alimentos que visa à diminuição do uso de conservantes sintéticos em seus produtos. Deste modo, sugere-se para trabalhos futuros, avaliar as combinações entre óleos e conservantes 
sintéticos in situ para verificar se esses efeitos serão mantidos ao interagirem com as moléculas dos alimentos, bem como avaliar o possível sinergismo entre os óleos e extratos vegetais, além da interação entre extratos vegetais e conservantes frente a bactérias deteriorantes, aumentando as possibilidades para a indústria de alimentos.

\section{Agradecimentos}

Os autores agradecem ao coordenador do Laboratório de Biotecnologia de Alimentos (Labiotec), do Departamento de Tecnologia de Alimentos (DTA-UFS) pelos auxílios prestados durante a execução do trabalho, a COOPAIBA/SE por ceder gentilmente os antimicrobianos sintéticos utilizados no estudo e ao CNPQ pelo financiamento da pesquisa.

\section{Referências}

Aligianis, N., Kalpoutzakis, E., Mitaku, S., \& Chinou, I. B. (2001). Composition and antimicrobial activity of the essential oil of two Origanum species. Journal of Agricultureand Food Chemistry, 49, 4168-4170. doi: 10.1021/jf001494m.

Alves, E. G., Vinholis, A. H. C., Casemiro, L. A., Furtado, N. A. J. C., Silva, M. L. A., Cunha, W. R., \& Martins, C. H. G. (2008). Estudo comparativo de técnicas de screening para avaliação da atividade anti-bacteriana de extratos brutos de espécies vegetais e de substâncias puras. Química Nova, 31(5), 12241229. doi:10.1590/s0100-40422008000500052

Amparo, T. R., Braga, V. C. C., Seibert, J. B., Souza, G. D., \& Teixeira, L. F. M. (2018). Métodos para avaliação in vitro da atividade antimicrobiana de plantas medicinais: a necessidade da padronização. Infarma, 30(1), 50-9. doi:10.14450/2318-9312.v30.e1.a2018.pp50-59

Bakkali, F., Averbeck, S., Averbeck, D., \& Idaomar, M. (2008). Biological effects of essential oils - A review. Food and Chemical Toxicology, 46,446 - 475. doi:10.1016/j.fct.2007.09.106

Bassolé, I. H. N., \& Juliani, H. R. (2012). Essential Oils in Combination and Their Antimicrobial Properties. Molecules, 17(4), 3989-4006. doi: 10.3390/molecules17043989

Brasil. (1998). Agência Nacional de Vigilância Sanitária - ANVISA. Portaria no 540 - SVS/MS de 27 de outubro de 1997. Aprova o Regulamento Técnico: Aditivos Alimentares - definições, classificação e emprego. http://antigo.anvisa.gov.br/legislacao\#/visualizar/382062.

Brasil. (2013). Agência Nacional de Vigilância Sanitária - ANVISA. Resolução - RDC noo 08, de 06 mar. de 2013. Dispõe sobre a aprovação de uso de aditivos alimentares para produtos de frutas e de vegetais e geleia de mocotó. Diário Oficial da União, Brasília.

Carvalho, I. O. (2016). Atividade antibacteriana de óleos essenciais e cremes dentais fitoterápicos em bactérias cariogênicas. Dissertação de Mestrado, Universidade Federal de Viçosa.https://locus.ufv.br//handle/123456789/9362

Castorani, G. M., \& Figueiredo, L. A. (2018). Atividade antimicrobiana in vitro de óleos essenciais contra patógenos alimentares. Trabalho de Conclusão de Curso do curso de Farmácia da Universidade Federal de Alfenas - MG.

CDC - Centers for Disease Control and Prevention. Food bornegerm sandillnesses. (2020). Disponível em: https://www.cdc.gov/foodsafety/foodbornegerms.html. Acessado em 02 de fevereiro de 2022.

Chen, H., \& Zhong, Q. (2018). Antibacterial activity of acidified sodium benzoate against Escherichia coli O157:H7, Salmonella enterica, and Listeria monocytogenes in tryptic soy broth and on cherry tomatoes. International Journal of Food Microbiology, 274, 38-44. doi: 10.1016/j.ijfoodmicro.2018.03.017

Chouhan, S., Sharma, K., \& Guleria, S. (2017). Antimicrobial Activity of Some Essential Oils-Present Status and Future Perspectives. Medicines (Basel, Switzerland), 4(3), 58. doi: 10.3390/medicines4030058

Copetti, N. F. (2019). Aditivos alimentares e suas consequências para a saúde humana. Trabalho de Conclusão de Curso de Graduação em Nutrição do Centro Universitário Unifacvest, Lages, Santa Catarina, 2019. https://bityli.com/WiEMR

Cutrim, E. S. M., Teles, A. M., Mouchrek, A. N., Mouchrek Filho, V. E., \& Everton, G. O. (2019). Avaliação da Atividade Antimicrobiana e Antioxidante dos Óleos Essenciais e Extratos Hidroalcoólicos de Zingiberofficinale (Gengibre) e Rosmarinusofficinalis (Alecrim) Rev. Virtual Quim., 11 (1), 60-81. doi: $10.21577 / 1984-6835.20190006$

Daltoé, M. M. L., Marques, C., Lise, C. C., Bordim, J.; Brenda, L. S., Casagrande, M., \& Lima, V. (2020). Percepção do consumidor sobre antioxidantes alimentares. sintético vs. natural. Avanços em Ciência e Tecnologia de Alimentos - Volume 1. https://downloads.editoracientifica.org/books/978-65-87196-589.pdf.

Ekhtelat, M., Bahrani, Z., Siahpoosh, A., \& Ameri, A. (2019). Evaluation of Antibacterial Effects of Mentha spicata L., Cuminum cyminum L. and Mentha longifolia L. Essential Oils Individually and in Combination with Sodium Benzoate Against Escherichia coli O157: H7 and Listeria monocytogenes. Jundishapur Journal Of Natural Pharmaceutical Products, 14(3). https://www.sid.ir/en/journal/ViewPaper.aspx?id=766109

Eninger, M., Ramos, A. V. G., \& Tiuman, T. S. (2017). Antibacterial activity of coriander and oregano essential oils and their effects on the association with chemical food preservative. $29^{\circ}$ Congresso Brasileiro de Microbiologia. Foz do Iguaçu, Paraná, Brasil. Disponível em: https://bityli.com/pXAHL Acessado em 02 de fevereiro de 2022 
Favero, D. M., Ribeiro, C. S. G., \& Aquino, A. D. (2011). Sulfitos: Importância na indústria alimentícia e seis possíveis malefícios à população. Segurança Alimentar e Nutricional, 18 (1), 11-20. doi: 10.20396/san.v18i1.8634684

Ferreira, R. D., Soares, R. A., Carvalho, L. A., Silva, G. A., Oliveira, C. A., \& Silva, G. A. (2020). Determinação da concentração inibitória mínima de conservantes alimentares para o controle de Salmonellatyphimurium. Holos, Ano 36, v.4. doi: 10.15628/holos.2020.10069

Fodil, S., Delgado, J., Varvaro, L., Yaseen, T., \& Rodríguez, A. (2018). Effect of potassium sorbate (E- 202) and the antifungal PgAFP protein on Aspergillus carbonariusgrowthand ochratoxin A production in raisin simulating media. Journal of the Science of Food and Agriculture, 98, 5785- 5794.doi: $10.1002 /$ jsfa. 9128

Freire, I. C.M., Pérez, A. L. A. L., Cardoso, A. M. R., Mariz, B. A. L. A., Almeida, L. F. D., Cavalcanti, Y. W., \& Padilha, W. W. N, (2014). Atividade antibacteriana de Óleos Essenciais sobre Streptococcus mutans e Staphylococcus aureus. Revista Brasileira de Plantas Medicinais, 16.doi:10.1590/1983084X/12_053

García-Díez, J., Alheiro, J. \& Pinto, A.L. (2016). Behaviour of food-borne pathogens on dry cured sausagemanufactured with herbs and spices essential oils andtheir sensorial acceptability. Food Control, 29, 262-270.doi:10.1111/jfpp.13056

Guimarães, A. L., Cunha, E. A., Matias, F. O., Garcia, P. G., Danopoulos, P., Swikidisa, R., Pinheiro, V. A., \& Nogueira, R. J. (2016). Antimicrobial activity of copaiba (Copaifera officinalis) and pracaxi (Pentaclethramacroloba) oils against staphylococcus aureus: importance in compounding for wound care. International Journal of Pharmaceutical Compounding, 20 (1), 58-62.

Gutierrez, J., Barry-Ryan, C., \& Bourke, P. (2008). The antimicrobial efficacy of plant essential oil combinations and interactions with food ingredients. International Journal of Food Microbiology, 124, 91-97. doi: 10.1016/j.ijfoodmicro.2008.02.028

Jay, J. M. (2005). Microbiologia de Alimentos. Artmed, $6^{\circ}$ edição.

Jing-En,L., Song-Tao, F., Zeng-Hui, Q., Chang, L, \& Shao-Ping, N. (2015). Total flavonoids content, antioxidant and antimicrobial activities of extracts from MoslachinensisMaxim. cv. Jiangxiangru.LWT -Food Science and Technology, 64(2), 1022-1027.doi: 10.1016/j.lwt.2015.07.033

Ladeira, N. M., Soares, R. A., Silva, E. M. C., Borges, S. V., Dias, M. V., Piccoli, R. H., \& Azevedo, V. M. (2017). Caracterização de filmes antimicrobianos a base de isolado proteico de soro/metabissulfito de sódio. Anais do 14 ${ }^{o}$ Congresso Brasileiro de Polímeros. http://edemocracia.com.br/cbpol/anais/2017/pdfs/plenary/01-007.pdf.

Lewis, R. E., Dickema, D. J., Messer, S. A., Pfaller, M. A., \& Klepser, M. E. (2002). Comparison of Estest, chequerboard dilution and time-kill studies for the detection of synergy or antagonism between antifungal agents tested against Candida species. Journal of Antimicrobial Chemotherapy, 49, 345-351. doi: $10.1093 / \mathrm{jac} / 49.2 .345$

Marchioro, P. N. C., Mariscal, A. G., Pinheiro, C., Nepoceno, K. L., Coelho, R. S. V. C., Fernandes, H. M., \& Vivi, V. K. (2018). Antimicrobial activity of natural/commercial copaiba oil-resin against standard strains. Journal Health NPEPS, 3 (2),527-539. doi: 10.30681/25261010

Masson, D. S., Salvador, S. L., Polizello A. C. M., \& Frade, M. A. C. (2013). Atividade antimicrobiana do óleo-resina de copaíba (Copaiferalangsdorfii) em bactérias de significância clínica em úlceras cutâneas. Revista Brasileira de Plantas Medicinais. 15 (4), 664-669. doi: 10.1590/S1516-05722013000500006

Mendonça, F. P. T. (2018). Atividade antimicrobiana de óleo essencial de manjericão (OcimumBasilicum l.) contra bactérias patogênicas e deterioradoras relacionadas a alimentos. Trabalho de Conclusão de Curso, Universidade Tecnológica Federal de Sergipe, São Cristovão, SE, Brasil. https://ri.ufs.br/handle/riufs/9638.

Ministério da Saúde. (2019).Surtos de doenças transmitidas por alimentos no Brasil - Informe 2018. Disponível em: https://bityli.com/KMiNr. Acessado em: 02 de fevereiro de 2022.

Mira, N. P., Teixeira, M. C., \& Sá-Correia, I. (2010). Adaptive response and tolerance toweak acids in Saccharomyces cerevisiae: a genome-wide view. Omics: a journal of integrative biology, 14 (5), 525-540. doi: 10.1089/omi.2010.0072

Monteiro, A. R. P. (2015). Atividade antimicrobiana de óleos essenciais. Trabalho de Conclusão de Curso. Universidade Fernando Pessoa, Porto. http://hdl.handle.net/10284/5327

Mothana, R. A., \& Lindequist, U. (2005). Antimicrobial activity of some medicinal plants of the island Soqotra. Journal of ethnopharmacology, 96 (1), 177181.doi:10.1016/j.jep.2004.09.006

NCCLS, National Committee for Clinical Laboratory Standards. (2003). Methods for Dilution Antimicrobial Susceptibility Tests for BacteriaThat Grow Aerobically; Approved Standard, Sixth Edition, M7-A6.

Oliveira, R. D. F. (2015). Incorporação de óleo essencial de manjericão em filmes biodegradáveis à base de galactomanana e óleo de canola. Dissertação de Mestrado, Universidade Federal do Ceará, Fortaleza - CE. https://repositorio.ufc.br/handle/riufc/16865

Pereira, A. S., Shitsuka, D. M., Parreira, F. J., \& Shitsuka, R. (2018). Metodologiada pesquisa científica. [e-book]. Santa Maria. Ed. UAB/NTE/UFSM. Recuperado de https://repositorio.ufsm.br/bitstream/handle/1/15824/Lic_Computacao_MetodologiaPesquisa-Cientifica.pdf?sequence=1

Pereira, L. F. S., Inácio, M. L. C., Pereira, R. C., \& De Angelis-Pereira, M. C. (2015). Prevalence of Additives in Processed Food Marketed in a South City of Minas Gerais. Revista Ciências Em Saúde, 5(3), 46-52. https://doi.org/10.21876/rcsfmit.v5i3.381. 
Ramos, A. V. G., Eninger, M.C., Tiuman, T. S., \& Adamczuck, S. S. (2015). Atividade antibacteriana e citotóxica do óleo essencial de tomilho (Thymusvulgaris l.) e avaliação de efeito sinérgico com conservantes sintéticos de alimentos. I Mostra Científica De Alimentos, Universidade Tecnológica Federal Do Paraná, Campus Medianeira. https://bityli.com/GJHsk.

Raut, J.S., \& Karuppayil, S.M. (2014). A status review on the medicinal properties of essential oils. Industrial Crops and Products, 62, 250 - 264. doi: 10.1016/j.indcrop.2014.05.055

Reis, J. B., Figueredo, L. A., Castorani, G. M., \& Veiga, S. M. O. M. (2020). Avaliação da atividade antimicrobiana dos óleos essenciais contra patógenos alimentares. Brazilian. Journal of Health Review.3 (1),342-363. doi: 10.34119/bjhrv3n1-025

Rodrigues, F. A. F. (2017). Avaliação da atividade antibacteriana e antibiofilme in vitro de óleos essenciais em Actinobacillus pleuropneumoniae. Dissertação de Mestrado, Universidade Federal de Viçosa, Viçosa. https://locus.ufv.br//handle/123456789/16537

Santos, A. O., Freire, J. A. S., Carvalho, T. D., Barbosa, T. C., Prates, R. P., Silva, J. C. R. L., \& Farias, P. K. S. (2016). Antibacterial and antioxidant activity of essential oils citrus with potentiality for inclusion as additives in foods. Caderno de Ciências Agrárias, 8 (3), 15-21.

Silva, A. A., Anjos, M. M., Ruiz, S. P., Panice, L. B., Mikcha, J. M. G., Junior, M. M., \& Filho, B. A. A. (2015). Avaliação da atividade antimicrobiana dos óleos essenciais de Thymusvulgaris (tomilho), Syzygium aromaticum (cravo-da-índia) e Rosmarinus officinalis (alecrim) e dos conservantes benzoato de sódio e sorbato de potássio em Escherichia coli e Staphylococcus aureus. B.ceppa, 33 (1), 111-117. doi: 10.5380/cep.v33i1.43814

Silva, A. C., Iacuzio, R., Silva C., Xavier, T. J. R. M., \& Cirone, N. C. S. (2018). Resistência antimicrobiana de Salmonella spp., Staphylococcus aureus e Escherichia coli isolados de carcaças de frangos: resistência a antibióticos e óleos essenciais. Revista Brasileira De Agropecuária Sustentável, 8(1). doi: $10.21206 /$ rbas.v8i1.474

Silva, A. J., Oliveira, L. P., Rezende, J. C. D. P., \& Saraiva, I. S. (2019). Avaliação da atividade antimicrobiana de óleos essenciais obtidos de diferentes fabricantes. Sinapse Múltipla, 8(1), 33-40. http://periodicos.pucminas.br/index.php/sinapsemultipla/article/view/19108.

Silva, K. B., \& Mello P. L. (2021). Antibacterial activity of the essential oils of pepper mint (Mentha Piperita), japanese mint (Mentha Arvensis) and basil (OcimumBasilicum) against salmonella enterica and staphylococcus aureus atcc. Revista Saúde,15 (3-4). doi: 10.33947/1982-3282-v15n3-4-4374

Spisni E., Petrocelli, G., Imbesi, V., Spigarelli, R., Azzinnari, D., Sarti, M. D., Campieri, M., \& Valerii, M. C. (2020). Antioxidant, Anti-Inflammatory, and Microbial-Modulating Activities of Essential Oils: Implications in Colonic Pathophysiology. Journalof Molecular Sciences. doi:10.3390/ijms21114152.

Tobouti, P. L., de Andrade Martins, T. C., Pereira, T. J., \& Mussi, M. C. M. (2017). Antimicrobial activity of copaiba oil: A review and a call for further research. Biomedicine \& Pharmacotherapy, 94, 93-99. doi:10.1016/j.biopha.2017.07.092

Wang, J., Ma, M., Yang, J., Chen, L., Yu, P., Wang, J., Gong, D., Deng, S., Wen, X., \& Zeng, Z. (2018). In vitro antibacterial activity and mechanism of monocaprylin against Escherichia coli and Staphylococcus aureus. Journal of Food Protection, 81, 1988-1996. doi: 10.4315/0362-028X.JFP-18-248

Zago, J. A. A., Ushimaru, P. I., Barbosa, L. N., \& Fernandes, A. J. (2009). Synergism between essential oils and antimicrobial drugs against Staphylooccus aureus and Escherichia coli strains from human infections. Revista Brasileira de Farmacognosia, 19 (4), 828-833. doi: 10.1590/S0102-695X2009000600005 